\title{
Macroscopic Models for Ionization in the Presence of Strong Electric Fields
}

\author{
P. Degond \\ A. Nouri \\ C. Schmeiser
}

Vienna, Preprint ESI 634 (1998)

November 27, 1998

Supported by Federal Ministry of Science and Transport, Austria Available via http://www.esi.ac.at 


\title{
Macroscopic Models for Ionization in the Presence of Strong Electric Fields *
}

\author{
P. Degond ${ }^{\dagger}$, A. Nouri ${ }^{\ddagger}$, and C. Schmeiser ${ }^{\S}$
}

\begin{abstract}
Starting from a kinetic model for electrons in a weakly ionized plasma, a spherical harmonic expansion (SHE-) model is derived by a formal asymptotic analysis for strongly disparate masses of electrons and ions. The macroscopic limit of the SHE-model for high fields gives a high field extension of standard macroscopic models for ionization.
\end{abstract}

\section{Introduction}

We consider the following scaled kinetic equation for electrons in a medium of ions and neutral particles, subject to ionization and recombination reactions:

$$
\varepsilon^{2} \frac{\partial f}{\partial t}+\varepsilon v \cdot \nabla_{x} f-\varepsilon E \cdot \nabla_{u} f=\varepsilon^{2} \tilde{Q}_{i o n}(f)+\tilde{Q}_{c o l l}(f),
$$

where $f(x, v, t), x, v \in \mathbb{R}^{3}, t \in \mathbb{R}$, is the electron distribution function, $E(x, t)$ is the (given) electric field, and the dimensionless parameter $\varepsilon^{2} \ll 1$ is the ratio of the electron mass to the mass of a neutral particle. The length scale has been chosen such that $\varepsilon$ can also be interpreted as the Knudsen

*This work has been supported by the TMR-network 'Asymptotic Methods in Kinetic Theory' and by the program on 'Charged Particle Kinetics' at the Erwin-SchrödingerInstitute, Vienna. The work of C.S. has also been supported by the Austrian Science Foundation under grant No. P11308-MAT.

† Mathématiques pour l'Industrie et la Physique, Université Paul Sabatier Toulouse 3, 118 route de Narbonne, 31062 Toulouse cedex, France.

$\ddagger$ UMR 5585, INSA, 20 av. A. Einstein, 69621 Villeurbanne Cedex, France.

$\S$ Institut für Angewandte und Numerische Mathematik, TU Wien, Wiedner Hauptstraße 8-10, 1040 Wien, Austria. 
number, i.e., the ratio of the mean free path (of an electron between (nonionizing) binary collisions with neutral particles) to the length scale. For the time scale a diffusion scaling has been chosen, which the following analysis will show to be appropriate (instead of a hydrodynamic scaling where $\varepsilon$ instead of $\varepsilon^{2}$ appears in front of the time derivative).

The terms $\tilde{Q}_{i o n}(f)$ and $\tilde{Q}_{\text {coll }}(f)$ describe the effects of ionization reactions and of the above mentioned binary collisions, respectively. A basic scaling assumption is that the mass ratio $\varepsilon^{2}$ is of the same order of magnitude as the ratio of typical time scales for collisions and ionization reactions. For the derivation of (1.1) and for further details of the scaling see [9].

Neutral particles and ions are assumed to be in local equilibrium, with the distribution functions given by the Maxwellians $f^{n}=M_{\rho_{n}, u, T}$ and $f^{i}=$ $M_{\rho_{i}, u, T}$, respectively, with

$$
M_{\rho, u, T}(v)=\frac{\rho}{(2 \pi T)^{3 / 2}} \exp \left(-\frac{|v-u|^{2}}{2 T}\right),
$$

and given space and time dependent $\rho_{n}, \rho_{i}, T \in \mathbb{R}$ and $u \in \mathbb{R}^{3}$. This can be derived [9] by assuming that the typical densities of electrons and ions are much smaller than those of neutral particles. Then, the dynamics of ions and neutral particles are dominated by binary collisions between neutral particles with each other as well as between neutral particles and ions. Therefore, both species are in local equilibrium with the same mean velocity and temperature.

We consider an impact ionization process where an highly energetic electron hits a neutral particle knocking out an additional electron and leaving back an ion. The reverse process is also considered. The ionization operator is then given by

$$
\begin{aligned}
\tilde{Q}_{i o n}(f)(v)= & \int_{\mathrm{R}^{12}}\left(f_{1}^{i} f_{2} f_{3}-f_{5}^{n} f\right) d \mu_{1235} \\
& +2 \int_{\mathrm{R}^{12}}\left(f_{5}^{n} f_{4}-f_{1}^{i} f_{3} f\right) d \mu_{1345},
\end{aligned}
$$

with the abbreviation

$$
\begin{aligned}
& d \mu_{1235}=\sigma_{i o n}\left(v_{1}, v_{2}, v_{3} \rightarrow v, v_{5}\right) \delta\left(\left(1-\varepsilon^{2}\right) v_{1}+\varepsilon\left(v_{2}+v_{3}-v\right)-v_{5}\right) \\
& \quad \times \delta\left(\left(1-\varepsilon^{2}\right)\left|v_{1}\right|^{2}+\left|v_{2}\right|^{2}+\left|v_{3}\right|^{2}-|v|^{2}-\left|v_{5}\right|^{2}+2 \Delta\right) d v_{1} d v_{2} d v_{3} d v_{5}
\end{aligned}
$$

and a similar definition of $d \mu_{1345}$, with $v_{2}$ and $v$ replaced by $v$ and $v_{4}$, respectively, and $d v_{2}$ replaced by $d v_{4}$. The support of the first Delta-distribution 
represents momentum conservation, and the second describes the loss of energy $(\Delta)$ in the ionization process. In (1.2), the subscript $j$ on a distribution function means evaluation at $v_{j}$. The ionization rate is assumed to be translation invariant:

$$
\begin{array}{r}
\sigma_{i o n}\left(v_{1}+\bar{v}, v_{2}+\varepsilon \bar{v}, v_{3}+\varepsilon \bar{v} \rightarrow v_{4}+\varepsilon \bar{v}, v_{5}+\bar{v}\right) \\
=\sigma_{i o n}\left(v_{1}, v_{2}, v_{3} \rightarrow v_{4}, v_{5}\right),
\end{array}
$$

for every $\bar{v} \in \mathbb{R}^{3}$. The appearance of the parameter $\varepsilon$ in the above equation is due to the fact that the electron velocities $\left(v_{2}, v_{3}, v_{4}\right)$ have been scaled differently from the ion and neutral particle velocities $\left(v_{1}, v_{5}\right)$.

Models of the form (1.2) for ionization and recombination phenomena are studied in [8], where an existence theorem is proven.

The binary collisions between electrons and neutral particles are modelled by

$$
\tilde{Q}_{c o l l}(f)(v)=\sigma_{\text {coll }} \int_{\mathrm{R}^{3}} \int_{\left(v-\varepsilon v_{*}\right) \cdot \Omega>0}\left|v-\varepsilon v_{*}\right|\left(f_{*}^{n \prime} f^{\prime}-f_{*}^{n} f\right) d \Omega d v_{*},
$$

with $0<\sigma_{\text {coll }} \in \mathbb{R},|\Omega|=1$, and

$$
\begin{aligned}
v^{\prime} & =v-\frac{2}{1+\varepsilon^{2}}\left(\left(v-\varepsilon v_{*}\right) \cdot \Omega\right) \Omega, \\
v_{*}^{\prime} & =v_{*}+\frac{2 \varepsilon}{1+\varepsilon^{2}}\left(\left(v-\varepsilon v_{*}\right) \cdot \Omega\right) \Omega .
\end{aligned}
$$

The subsequent computations are greatly simplified by the coordinate transformation $v-\varepsilon u \rightarrow v$. The transformed version of equation (1.1) is given by

$$
\begin{aligned}
\varepsilon^{2} \frac{\partial f}{\partial t} & -\varepsilon^{3} \frac{\partial u}{\partial t} \cdot \nabla_{v} f+\varepsilon(v+\varepsilon u) \cdot\left(\nabla_{x} f-\varepsilon \nabla_{x} u \nabla_{v} f\right)-\varepsilon E \cdot \nabla_{v} f \\
& =\varepsilon^{2} Q_{\text {ion }}(f)+Q_{\text {coll }}(f)
\end{aligned}
$$

where $Q_{\text {ion }}(f)$ is defined by (1.2) with $f^{n}$ and $f^{i}$ replaced by $M_{\rho_{n}, 0, T}$ and $M_{p_{i}, 0, T}$, respectively. Similarly, $Q_{\text {coll }}(f)$ is defined by (1.3) with $f^{n}$ replaced by $M_{\rho_{n}, 0, T}$. Thus, in (1.5) the mean velocity of the background medium appears in the transport operator instead of the ionization and collision operators.

In the following section some results on the asymptotics of the collision operator $Q_{\text {coll }}$ as $\varepsilon \rightarrow 0$ are presented. This is an extension of some of the 
results of [3]. Section 3 contains the formal asymptotics as $\varepsilon \rightarrow 0$ for solutions of (1.5). In the limit, the electron distribution function is approximated by an isotropic distribution satisfying a diffusion equation with both spatial diffusion as well as diffusion in the energy direction. Related results can be found in [6]. The limiting equation is an example of a SHE model, typical for situations where the dominating collision mechanism is (almost) elastic. This situation occurs frequently in semiconductor physics [1], [5], [11]. For hydrodynamic limits of disparate mass binary gases under different scaling assumptions see [4].

Finally, in section 4, a macroscopic limit of the SHE model is computed under the scaling assumption that the influence of the electric field balances that of the inelastic contributions to binary collisions. The equilibrium distribution is field dependent and interpolates between the Maxwellian distribution for low fields and the Druyvenstein distribution for low temperatures. The resulting equation for the macroscopic density contains field dependent transport parameters and ionization rates. A related treatment for the transport in semiconductors can be found in [2].

\section{Properties of the collision operator}

Before carrying out the limit $\varepsilon \rightarrow 0$ in (1.5) we collect some properties of the collision operator

$$
Q_{\text {coll }}(f)(v)=\sigma_{\text {coll }} \int_{\mathrm{R}^{3}} \int_{\left(v-\varepsilon v_{*}\right) \cdot \Omega>0}\left|v-\varepsilon v_{*}\right|\left(f^{\prime} M_{*}^{\prime}-f M_{*}\right) d \Omega d v_{*},
$$

where here and in the following $M=M_{\rho_{n}, 0, T}$.

Assuming a formal expansion of a solution of (1.5) in the form $f=$ $f_{0}+\varepsilon f_{1}+\varepsilon^{2} f_{2}+O\left(\varepsilon^{3}\right)$, and letting $\varepsilon \rightarrow 0$ in (1.5) we obtain

$$
Q_{0}\left(f_{0}\right):=\sigma_{\text {coll }} \rho_{n}|v| \int_{v \cdot \Omega>0}\left(\hat{f}_{0}-f_{0}\right) d \Omega=0,
$$

where denotes evaluation at $\hat{v}=v-2(v \cdot \Omega) \Omega$, the $(\varepsilon=0)$-version of the postcollisional velocity $v^{\prime}$. It has been shown in [3] that the kernel of the elastic collision operator $Q_{0}$ consists of the functions depending on $v$ only through the energy $w=|v|^{2} / 2$. Thus, $f_{0}=f_{0}(w)$ holds. Note that in this

section we shall suppress the dependence of the distribution function on $x$ 
and $t$. The velocity will frequently be written in terms of energy and angular coordinates: $v=\omega \sqrt{2 w}, \omega \in S^{2}$.

Another important property of $Q_{0}$ is its symmetry with respect to the $L^{2}\left(\mathbb{R}^{3}\right)$ inner product:

$$
\int_{\mathbf{R}^{3}} Q_{0}(f) g d v=-\frac{\sigma_{\text {coll } \rho_{n}}}{2} \int_{\mathrm{R}^{3}}|v| \int_{\nu \cdot \Omega>0}(\hat{f}-f)(\hat{g}-g) d \Omega d v .
$$

The collision invariants of $Q_{0}$ are all functions of the energy $w$, implying

$$
\int_{S^{2}} Q_{0}(f) d \omega=0
$$

For an $\varepsilon$-independent argument $f$, the collision operator can be expanded in the form $Q_{\text {coll }}(f)=Q_{0}(f)+\varepsilon Q_{1}(f)+\varepsilon^{2} Q_{2}(f)+O\left(\varepsilon^{3}\right)$. Then, for a solution of (1.5) we have

$$
\begin{aligned}
Q_{\text {coll }}(f)= & \varepsilon\left(Q_{0}\left(f_{1}\right)+Q_{1}\left(f_{0}\right)\right) \\
& +\varepsilon^{2}\left(Q_{0}\left(f_{2}\right)+Q_{1}\left(f_{1}\right)+Q_{2}\left(f_{0}\right)\right)+O\left(\varepsilon^{3}\right) .
\end{aligned}
$$

It is the main objective of this section to compute information on the terms in this expansion.

First we collect some basic results, the proofs of which follow from straightforward computations:

Lemma 2.1 i) For any $\Omega \in S^{2}$,

$$
\int_{\mathbf{R}^{3}} M_{*}\left(v_{*} \cdot \Omega\right)^{2} d v_{*}=\rho_{n} T .
$$

ii) For any $v \in \mathbb{R}^{3}$,

$$
\int_{v \cdot \Omega>0}(v \cdot \Omega)^{2} d \Omega=\frac{2 \pi}{3}|v|^{2} .
$$

Lemma 2.2 Formal computations give $Q_{1}\left(f_{0}\right)=0$ and

$$
\begin{aligned}
& \sqrt{2 w} \int_{S^{2}}\left[Q_{1}\left(f_{1}\right)+Q_{2}\left(f_{0}\right)\right] d \omega=\sqrt{2 w} \int_{S^{2}} Q_{2}\left(f_{0}\right) d \omega \\
& =\frac{64 \pi^{2}}{3} \sigma_{\text {coll }} \rho_{n} T \frac{\partial}{\partial w}\left[w^{2}\left(\frac{f_{0}}{T}+\frac{\partial f_{0}}{\partial w}\right)\right] .
\end{aligned}
$$


Proof. We start with a weak formulation of the collision term. With a test function of the form $\varphi=\varphi_{0}(w)+\varepsilon \varphi_{1}(v)$, we have

$$
\begin{aligned}
\int_{\mathrm{R}^{3}} Q_{\text {coll }}(f) \varphi d v= & \varepsilon \int_{\mathrm{R}^{3}} Q_{1}\left(f_{0}\right) \varphi_{0} d v+\varepsilon^{2} \int_{\mathrm{R}^{3}}\left[Q_{0}\left(f_{1}\right)+Q_{1}\left(f_{0}\right)\right] \varphi_{1} d v \\
& +\varepsilon^{2} \int_{\mathrm{R}^{3}}\left[Q_{1}\left(f_{1}\right)+Q_{2}\left(f_{0}\right)\right] \varphi_{0} d v+O\left(\varepsilon^{3}\right)
\end{aligned}
$$

On the other hand, we use the symmetry property

$$
\begin{aligned}
\int_{\mathrm{R}^{3}} Q_{\text {coll }}(f) \frac{g}{M} d v=-\frac{\sigma_{\text {coll }}}{2} \int_{\mathrm{R}^{6}} \int_{\left(v-\varepsilon v_{*}\right) \cdot \Omega>0}\left|v-\varepsilon v_{*}\right| M M_{*} \\
\\
\times\left(\frac{f^{\prime}}{M^{\prime}}-\frac{f}{M}\right)\left(\frac{g^{\prime}}{M^{\prime}}-\frac{g}{M}\right) d \Omega d v_{*} d v,
\end{aligned}
$$

with $g=g_{0}(w)+\varepsilon g_{1}(v)$. Using (1.4) we expand

$$
\begin{aligned}
& \frac{f^{\prime}}{M^{\prime}}-\frac{f}{M}=\frac{\varepsilon}{M}\left(F_{0}+F_{1}\right)+O\left(\varepsilon^{2}\right), \\
& F_{0}=-2\left(v_{*} \cdot \Omega\right)(v \cdot \Omega)\left(\frac{f_{0}}{T}+\frac{\partial f_{0}}{\partial w}\right), \quad F_{1}=\hat{f}_{1}-f_{1},
\end{aligned}
$$

implying

$$
\begin{aligned}
& \int_{\mathrm{R}^{3}} Q_{\text {coll }}(f) \frac{g}{M} d v \\
& =-\varepsilon^{2} \frac{\sigma_{\text {coll }}}{2} \int_{\mathrm{R}^{6}} \int_{v \cdot \Omega>0}|v| \frac{M_{*}}{M}\left(F_{0}+F_{1}\right)\left(G_{0}+G_{1}\right) d \Omega d v_{*} d v+O\left(\varepsilon^{3}\right) .
\end{aligned}
$$

With the notation

$$
A_{i j}=-\frac{\sigma_{\text {coll }}}{2} \int_{\mathrm{R}^{6}} \int_{v \cdot \Omega>0}|v| \frac{M_{*}}{M} F_{i} G_{j} d \Omega d v_{*} d v,
$$

(2.2) implies

$$
A_{11}=\int_{\mathbf{R}^{3}} Q_{0}\left(f_{1}\right) \frac{g_{1}}{M} d v .
$$

Also, $A_{10}=A_{01}=0$ holds since the Maxwellian has zero mean velocity and therefore the integral with respect to $v_{*}$ vanishes. Finally, in

$$
\begin{array}{r}
A_{00}=-2 \sigma_{\text {coll }} \int_{\mathbf{R}^{6}}|v| \frac{M_{*}}{M}\left(\frac{f_{0}}{T}+\frac{\partial f_{0}}{\partial w}\right)\left(\frac{g_{0}}{T}+\frac{\partial g_{0}}{\partial w}\right) \\
\int_{v \cdot \Omega>0}\left(v_{*} \cdot \Omega\right)^{2}(v \cdot \Omega)^{2} d \Omega d v_{*} d v,
\end{array}
$$


we use Lemma 2.1:

$$
A_{00}=-\frac{4 \pi}{3} \sigma_{\text {coll }} \rho_{n} T \int_{\mathrm{R}^{3}}|v|^{3}\left(\frac{f_{0}}{T}+\frac{\partial f_{0}}{\partial w}\right) \frac{\partial}{\partial w}\left(\frac{g_{0}}{M}\right) d v .
$$

After the introduction of energy and angular variables and an integration by parts this becomes

$$
A_{\mathrm{oo}}=\frac{64 \pi^{2}}{3} \sigma_{\text {coll }} \rho_{n} T \int_{0}^{\infty} \frac{\partial}{\partial w}\left[w^{2}\left(\frac{f_{0}}{T}+\frac{\partial f_{0}}{\partial w}\right)\right] \frac{g_{0}}{M} d w .
$$

Comparison of

$$
\int_{\mathrm{R}^{3}} Q_{c o l l}(f) \frac{g}{M} d v=\varepsilon^{2}\left(A_{00}+A_{11}\right)+O\left(\varepsilon^{3}\right)
$$

for $g=\varphi M$ with (2.3) completes the proof.

\section{The diffusion approximation}

In this section we carry out the formal limit $\varepsilon \rightarrow 0$ in the transport equation

(1.5). Substituting the ansatz $f=f_{0}+\varepsilon f_{1}+\varepsilon^{2} f_{2}+O\left(\varepsilon^{3}\right)$ in (1.5) and comparing coefficients of powers of $\varepsilon$ gives

$$
\begin{aligned}
& 0=Q_{0}\left(f_{0}\right) \\
& v \cdot \nabla_{x} f_{0}-E \cdot \nabla_{v} f_{0}=Q_{0}\left(f_{1}\right)+Q_{1}\left(f_{0}\right) \\
& \frac{\partial f_{0}}{\partial t}+u \cdot \nabla_{x} f_{0}-v^{t r} \nabla_{x} u \nabla_{v} f_{0}+v \cdot \nabla_{x} f_{1}-E \cdot \nabla_{v} f_{1} \\
& \quad=Q_{\text {ion }}^{0}\left(f_{0}\right)+Q_{0}\left(f_{2}\right)+Q_{1}\left(f_{1}\right)+Q_{2}\left(f_{0}\right) .
\end{aligned}
$$

The ionization operator $Q_{i o n}^{0}$ is obtained by setting $\varepsilon=0$ in $Q_{\text {ion }}$ :

$$
\begin{aligned}
& Q_{i o n}^{0}(f) \\
& =\int_{\mathrm{R}^{6}} \sigma_{i o n}^{0}\left(v_{2}, v_{3} \rightarrow v\right) \delta\left(w_{2}+w_{3}+\Delta-w\right)\left(\rho_{i} f_{2} f_{3}-\rho_{n} f\right) d v_{2} d v_{3} \\
& +2 \int_{\mathrm{R}^{6}} \sigma_{i o n}^{0}\left(v, v_{3} \rightarrow v_{4}\right) \delta\left(w+w_{3}+\Delta-w_{4}\right)\left(\rho_{n} f_{4}-\rho_{i} f f_{3}\right) d v_{3} d v_{4},
\end{aligned}
$$

with $w=|v|^{2} / 2, w_{j}=\left|v_{j}\right|^{2} / 2, j=2,3,4$, and

$$
\sigma_{i o n}^{0}\left(v_{2}, v_{3} \rightarrow v_{4}\right)=\int_{\mathrm{R}^{3}} \sigma_{i o n}\left(v_{1}, v_{2}, v_{3} \rightarrow v_{4}, v_{1}\right) M_{1,0, T}\left(v_{1}\right) d v_{1}
$$


The results of the previous section and the first equation imply $f_{0}=$ $f_{0}(x, w, t)$. Also, by Lemma 2.2, the second equation can be written as

$$
v \cdot\left(\nabla_{x} f_{0}-E \frac{\partial f_{0}}{\partial w}\right)=Q_{0}\left(f_{1}\right) .
$$

A straightforward computation using (2.1) shows that the general solution is given by

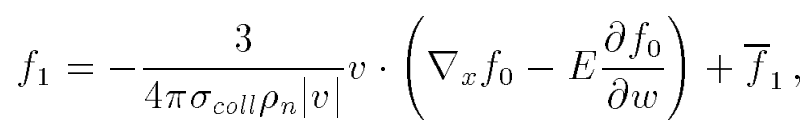

with $\bar{f}_{1}=\bar{f}_{1}(x, w, t)$. Finally, an equation for the determination of $f_{0}$ is obtained by integrating (3.1) over balls. As a preliminary computation, it is easily shown that

$$
\begin{aligned}
& \sqrt{2 w} \int_{S^{2}}\left(u \cdot \nabla_{x} f_{0}-v^{t r} \nabla_{x} u \nabla_{v} f_{0}\right) d w \\
& =\nabla_{x} \cdot\left(4 \pi \sqrt{2 w} u f_{0}\right)-\frac{\partial}{\partial w}\left(\frac{4 \pi}{3}(2 w)^{3 / 2}\left(\nabla_{x} \cdot u\right) f_{0}\right)
\end{aligned}
$$

holds. Then, the above mentioned integration of (3.1) with lemma 2.2 gives

$$
\begin{aligned}
& \sqrt{2 w} \frac{\partial f_{0}}{\partial t}+\nabla_{x} \cdot\left(\sqrt{2 w} u f_{0}\right)-\frac{\partial}{\partial w}\left(\frac{1}{3}(2 w)^{3 / 2}\left(\nabla_{x} \cdot u\right) f_{0}\right)+\nabla_{x} \cdot j \\
& -\frac{\partial}{\partial w}(E \cdot j)=\frac{\partial}{\partial w}\left[\frac{16 \pi \sigma_{\text {coll }} \rho_{n} T}{3} w^{2}\left(\frac{f_{0}}{T}+\frac{\partial f_{0}}{\partial w}\right)\right]+\bar{Q}_{\text {ion }}\left(f_{0}\right)
\end{aligned}
$$

with

$$
4 \pi j:=\sqrt{2 w} \int_{S^{2}} v f_{1} d \omega=-\frac{2 w}{\sigma_{c o l l} \rho_{n}}\left(\nabla_{x} f_{0}-E \frac{\partial f_{0}}{\partial w}\right)
$$

and

$$
\begin{aligned}
\bar{Q}_{i o n}(f)= & \frac{\sqrt{2 w}}{4 \pi} \int_{S^{2}} Q_{i o n}^{0}(f) d w \\
= & \int_{0}^{\infty} \int_{0}^{\infty} \sqrt{w_{2} w_{3} w} \bar{\sigma}_{i o n}\left(w_{2}, w_{3} \rightarrow w\right) \\
& +2 \int_{0}^{\infty} \int_{0}^{\infty} \sqrt{w w_{3} w_{4}} \bar{\sigma}_{i o n}\left(w, w_{3} \rightarrow w_{4}\right) \\
& \delta\left(w+w_{3}+\Delta-w_{4}\right)\left(\rho_{n} f_{4}-\rho_{i} f f_{3}\right) d w_{3} d w_{4},
\end{aligned}
$$


where

$$
\begin{aligned}
& \bar{\sigma}_{i o n}\left(w_{2}, w_{3} \rightarrow w_{4}\right) \\
& =\frac{1}{\pi \sqrt{2}} \int_{\left(S^{2}\right)^{3}} \sigma_{i o n}^{0}\left(\omega_{2} \sqrt{2 w_{2}}, \omega_{3} \sqrt{2 w_{3}} \rightarrow \omega_{4} \sqrt{2 w_{4}}\right) d \omega_{2} d \omega_{3} d \omega_{4} .
\end{aligned}
$$

The derivation of (3.2) has been the aim of our formal asymptotics. The equation can also be written as

$$
\sqrt{2 w} \frac{\partial f_{0}}{\partial t}+\nabla_{x} \cdot J_{x}+\frac{\partial J_{w}}{\partial w}=\bar{Q}_{i o n}\left(f_{0}\right)
$$

with

$$
\begin{aligned}
J_{x}= & \sqrt{2 w} u f_{0}-\frac{w}{2 \pi \sigma_{\text {coll }} \rho_{n}}\left(\nabla_{x} f_{0}-E \frac{\partial f_{0}}{\partial w}\right) \\
J_{w}= & \frac{w}{2 \pi \sigma_{\text {coll } \rho_{n}}} E \cdot\left(\nabla_{x} f_{0}-E \frac{\partial f_{0}}{\partial w}\right)-\frac{(2 w)^{3 / 2}}{3}\left(\nabla_{x} \cdot u\right) f_{0} \\
& -\frac{16 \pi \sigma_{\text {coll } \rho_{n} T} T}{3} w^{2}\left(\frac{f_{0}}{T}+\frac{\partial f_{0}}{\partial w}\right) .
\end{aligned}
$$

Proposition 3.1 The operator on the left hand side of (3.4) is parabolic for $w>0$, but not uniformly parabolic.

Proof. A simple computation shows that the leading part of the second order operator in position-energy direction is represented by the quadratic form

$$
\frac{w}{2 \pi \sigma_{\text {coll }} \rho_{n}}|X-E W|^{2}+\frac{16 \pi \sigma_{\text {coll }} \rho_{n} T}{3} w^{2} W^{2}, \quad X \in \mathbb{R}^{3}, W \in \mathbb{R},
$$

which is positive definite, but not uniformly, for $w>0$.

The degeneracy of the equation at the energy boundary $w=0$ implies that the natural zero flux condition

$$
J_{w}=0, \quad \text { for } w=0
$$

is sufficient as a boundary condition. It only excludes too strong singularities of $f_{0}$ at $w=0$. 


\section{A Macroscopic Limit at High Fields}

In this section we assume that ionization effects are weak compared to the inelastic contribution of the collision effects. Starting with the diffusion model (3.4), we rewrite the ionization cross section $\bar{\sigma}_{i o n}$ as $\gamma \bar{\sigma}_{i o n}$ with a small dimensionless parameter $\gamma$. We are interested in effects occurring on length and time scales relevant for the ionization processes. Therefore, the rescaling $x \rightarrow x / \gamma, t \rightarrow t / \gamma$ is introduced in (3.4):

$$
\gamma \sqrt{2 w} \frac{\partial f_{0}}{\partial t}+\gamma \nabla_{x} \cdot J_{x}+\frac{\partial J_{w}}{\partial w}=\gamma \bar{Q}_{i o n}\left(f_{0}\right)
$$

with

$$
\begin{aligned}
J_{x}= & \sqrt{2 w} u f_{0}-\frac{w}{2 \pi \sigma_{\text {coll }} \rho_{n}}\left(\gamma \nabla_{x} f_{0}-E \frac{\partial f_{0}}{\partial w}\right) \\
J_{w}= & \frac{w}{2 \pi \sigma_{\text {coll } \rho_{n}}} E \cdot\left(\gamma \nabla_{x} f_{0}-E \frac{\partial f_{0}}{\partial w}\right)-\gamma \frac{(2 w)^{3 / 2}}{3}\left(\nabla_{x} \cdot u\right) f_{0} \\
& -\frac{16 \pi \sigma_{\text {coll } \rho_{n}} T}{3} w^{2}\left(\frac{f_{0}}{T}+\frac{\partial f_{0}}{\partial w}\right) .
\end{aligned}
$$

The ionization operator $\bar{Q}_{i o n}$ is given by (3.3).

In the formal limit $\gamma \rightarrow 0$, (4.1) reduces to

$$
\frac{\partial}{\partial w}\left(\left(A^{2} w+T w^{2}\right) \frac{\partial f_{0}}{\partial w}+w^{2} f_{0}\right)=0,
$$

with

$$
A(x, t)=\sqrt{\frac{3}{2}} \frac{|E(x, t)|}{4 \pi \sigma_{\text {coll }} \rho_{n}(x, t)} .
$$

The general solution satisfying the zero flux condition (3.5) is given by $f_{0}(x, w, t)=\rho_{e}(x, t) \mathcal{M}_{A(x, t), T(x, t)}(w)$ with arbitrary $\rho_{e}$ and

$$
\mathcal{M}_{A, T}(w)=c_{A, T} \exp \left(-\int_{0}^{w} \frac{s d s}{s T+A^{2}}\right),
$$

where $c_{A, T}$ is chosen such that

$$
\int_{0}^{\infty} \sqrt{2 w} \mathcal{M}_{A, T}(w) d w=1
$$


Limiting cases of the equilibrium distribution are the Maxwellian

$$
\mathcal{M}_{0, T}(w)=\sqrt{\frac{2}{\pi T^{3}}} \epsilon^{-w / T}
$$

and the Druyvenstein distribution [7]

$$
\mathcal{M}_{A, 0}(w)=\frac{1}{A^{3 / 2} 2^{1 / 4} \Gamma(3 / 4)} e^{-w^{2} /\left(2 A^{2}\right)} .
$$

An equation for the macroscopic electron density $\rho_{e}$ is obtained by integrating (4.1) with respect to $w$ :

$$
\frac{\partial \rho_{e}}{\partial t}+\nabla_{x} \cdot\left[\rho_{e}(u-\mu E)\right]=q_{\text {ion }}\left(\rho_{e}\right) .
$$

The mobility is given by

$$
\mu=\frac{1}{2 \pi \sigma_{\text {coll } \rho_{n}}} \int_{0}^{\infty} \mathcal{M}_{A, T}(w) d w,
$$

and the macroscopic ionization rate by

$$
q_{\text {ion }}\left(\rho_{e}\right)=\rho_{e}\left(a \rho_{n}-b \rho_{i} \rho_{e}\right),
$$

with

$$
\begin{aligned}
a(A, T, \Delta)= & \int_{0}^{\infty} \int_{0}^{\infty} \int_{0}^{\infty} \sqrt{w_{2} w_{3} w_{4}} \bar{\sigma}_{i o n}\left(w_{2}, w_{3} \rightarrow w_{4}\right) \\
& \delta\left(w_{2}+w_{3}+\Delta-w_{4}\right) \mathcal{M}_{A, T}\left(w_{4}\right) d w_{2} d w_{3} d w_{4}, \\
b(A, T, \Delta)= & \int_{0}^{\infty} \int_{0}^{\infty} \int_{0}^{\infty} \sqrt{w_{2} w_{3} w_{4}} \bar{\sigma}_{i o n}\left(w_{2}, w_{3} \rightarrow w_{4}\right) \\
& \delta\left(w_{2}+w_{3}+\Delta-w_{4}\right) \mathcal{M}_{A, T}\left(w_{2}\right) \mathcal{M}_{A, T}\left(w_{3}\right) d w_{2} d w_{3} d w_{4} .
\end{aligned}
$$

The equilibrium condition $\rho_{i} \rho_{e}=\frac{a}{b} \rho_{n}$ is a high field generalization of the Saha law [10]

$$
\rho_{i} \rho_{e}=\sqrt{\frac{\pi T^{3}}{2}} e^{-\Delta / T} \rho_{n},
$$

which is recovered for $A=0$. 


\section{References}

[1] N. Ben Abdallah, P. Degond, On a hierarchy of macroscopic models for semiconductors, J. Math. Phys. 37 (1996), pp. 3306-3333.

[2] N. Ben Abdallah, P. Degond, P.A. Markowich, C. Schmeiser, High field approximations of the spherical harmonics expansion model for semiconductors, manuscript, TU Wien, 1998.

[3] Degond, P., Lucquin-Desreux, B.. The asymptotics of collision operators for two species of particles of disparate mass, Math. Models Methods Appl. Sci. 6 (1996), pp. 405-436.

[4] P. Degond, B. Lucquin-Desreux, Transport coefficients of plasmas and disparate mass binary gases, Transp. Th. and Stat. Phys. 25 (1996), pp. $595-633$.

[5] P. Dmitruk, A. Saul, L. Reyna, High electric field approximation to charge transport in semiconductor devices, Appl. Math. Letters 5 (1992), pp. 99-102.

[6] J.L. Lebowitz, A. Rokhlenko, Hydrodynamical equation for electron swarms, preprint, Rutgers University, 1998.

[7] R.L. Liboff, Kinetic Theory, Classical Quantum and Relativistic Descriptions, Prentice Hall International, 1990.

[8] A. Nouri, A. de La Bourdonnaye, Ionization and recombination in plasmas, J. Stat. Phys. 87 (1997), pp. 643-660.

[9] A. Nouri, C. Schmeiser, A diffusion approximation of ionizing collisions in weakly ionized plasmas, in preparation.

[10] Y. Raizer, Gas Discharge Physics, Springer Verlag, 1994.

[11] C. Schmeiser, A. Zwirchmayr, Elastic and drift-diffusion limits of electron-phonon interaction in semiconductors, Math. Models and Meth. in Appl. Sci. 8 (1998), pp. 37-53. 